\title{
DE L'ABDUCTION CRÉATIVE COMME MÉTHODE SÉMIO-ANTHROPOLOGIQUE AU SERVICE DE LA SOCIOLOGIE DE LA CONNAISSANCE ET DES REPRÉSENTATIONS
}

\section{Florent Gaudez}

\author{
Les faits empêchent de voir la vérité. \\ Virginia Woolf \\ La vérité des faits, on ne la trouve pas en la \\ consommant mais en la produisant. \\ P. Macherey
}

\begin{abstract}
Résumé: Une œuvre d'art ne symbolise jamais du social ex nihilo, elle re-symbolise. Si l'on définit le travail de l'œuvre comme une resymbolisation du donné symbolique, la première étape sociologique face à une œuvre consiste à construire, à partir de matériaux culturels, la configuration du champ symbolique. Une telle posture ouvre des perspectives sur la sociologie des représentations mais aussi éclaire la complexité de l'œuvre d'art et la multiplicité de stratégies littéraires et cognitives propres à l'œuvre. La description sémiologique des structures de l'œuvre est l'une des méthodes les plus fécondes pour replacer l'œuvre dans son contexte historico-sociologique et soumettre à la vérification une étude sociologique. En nous appuyant sur les travaux de C.-S. Peirce et U. Eco, il s'agit ici d'indiquer quelques pistes de réflexion dans cette direction en proposant la démarche abductive qui, plus intuitive que le procès déductif, inventorie et cherche à relier, organiser des données, des signes qui lui semblent devoir découler d'une loi à advenir, mais encore inconnue.
\end{abstract}

Mots-clefs: abduction, œuvre d'art, Sémiologie, Sociologie, symbolique.

* Centre d'Étude des Rationalités et des Savoirs, PARS@C2-CERS (CNRS-UMR 5117), Pack Albigeois Rationalités-S@ voirs-Culture, Université de Toulouse 2 - Le Mirail - France.

Artigo recebido em 11 ago. 2004; aprovado em 8 out. 2004. 
Comme le rappelle J.-C. Passeron (1999): "Max Weber s'est escrimé à montrer que la 'neutralité axiologique' n'excluait pas, mais au contraire imposait au sociologue et à l'historien 'le rapport aux valeurs' comme instrument interprétatif dans la construction et la description des faits de valeur culturelle". La distinction que fait Weber (1992, p. 384) entre "rapport aux valeurs" et "évaluation" est fondamentale: "c'est par rapport au second terme que prend sens la "neutralité axiologique" [expression d'ailleurs toujours entre guillemets], entendue comme un refus "d'affirmer quoi que ce soit sur ce qui doit valoir" ou comme "l'exigence extrêmement triviale qui impose au savant ou au professeur de faire absolument la distinction, puisque ce sont deux séries de problèmes tout simplement hétérogènes, entre la constatation des faits empiriques (...) et $s a$ propre prise de position évaluative de savant qui porte un jugement sur des faits"( Idem, p. 380). Ainsi, Weber n' interdit pas complètement aux savants "d'exprimer sous forme de jugements de valeur les idéaux qui les animent" (Idem, p. 131), mais sous deux conditions:

- porter scrupuleusement, à chaque instant, à leur propre conscience et à celle des lecteurs quels sont les étalons de valeur qui servent à mesurer la réalité et ceux d'où ils font dériver le jugement de valeur;

- distinguer "clairement" ce qui relève de la "discussion scientifique des faits" et du "raisonnement axiologique. (Weber, 1994, p. 131132)

Lorsque la sociologie considère l'œuvre d' art (et pas seulement les producteurs de valeur, les stratégies de valorisation, les institutions légitimantes, etc.) elle fait comme si elle avait affaire à des comportements symbolisant la réalité sociale, c'est-à-dire la traduisant dans un langage (J. Leenhardt). Or, de même qu'une lutte sociale retravaille tout à la fois des rapports sociaux et des représentations, de même une œuvre d'art ne symbolise jamais du social ex nihilo, elle re-symbolise. Définir l'œuvre comme re-symbolisation, c'est comprendre que l'artiste y réfléchit, c'est-à-dire y travaille, dans des conditions sociales données, le rapport qui est pour lui le plus fondamental de ses rapports sociaux : son rapport à la langue, à son histoire linguistique ou littéraire (Leenhardt, 1999, p. 385-395). 
Si certains considèrent l'œuvre littéraire comme un simple document relatif à une période historique, d'autres préfèrent partir de l'élément social pour expliquer la solution esthétique; une troisième possibilité consiste à établir une dialectique entre ces deux points de vue, c'est-à-dire envisager l'œuvre comme fait esthétique, et la société comme contexte explicatif. Dans cette perspective, l'élément social détermine les choix esthétiques, mais l'étude de l'œuvre et de ses caractéristiques structurales permet de mieux comprendre la situation d'une société.

C'est là l'une des questions que pose U. Eco (1992, 1996): de quelle utilité peuvent être, dans ce dernier cas, les études sémiologiques portant sur les macrostructures de communication que représentent les éléments d'un système narratif et discursif?

Si la description de l'œuvre comme système de signes offrait la possibilité de mettre en évidence les structures signifiantes de cette œuvre d'une façon absolument neutre et objective (sans tenir compte de l'ensemble com-plexe des signifiés que l'histoire attribue continuellement à l'œuvre message), le contexte social lui-même et l'idéologie présente dans l'œuvre toute entière, considérée comme un signe global, demeureraient exclus, au moins partiellement de l'étude sémiologique. Il s'agit en fait d'une apparence, car il n'est possible d'identifier et de mettre en évidence un signifiant qu'en lui attribuant une signification. Ainsi, le fait d'extraire des structures signifiantes dans une œuvre, c'est déjà les poser comme étant particulièrement pertinentes relativement aux idées que l'on a l'intention de proposer sur cette œuvre, et c'est donc se situer dans une perspective interprétative. De ce fait, une analyse structurale des signes contenus dans l'œuvre relève automatiquement d'une vérification d'hypothèses historiques comme sociologiques.

Ainsi, pour U. Eco, la description sémiologique des structures de l'œuvre est l'une des méthodes les plus fécondes pour replacer l'œuvre dans son contexte historico-sociologique. C'est pourquoi il préconise qu'une étude sociologique ${ }^{1}$ soit soumise à la vérification sémiologique. 
Ce qu'il propose à cette fin est une méthode dite "circulaire" permettant de passer du contexte social (externe) au contexte structural (interne) de l'œuvre appréhendée. Il s'agit de construire une description de ces deux contextes ainsi que ceux introduits par le jeu interprétatif, selon des critères homogènes, et de mettre en évidence des homologies de structures entre ces contextes. En appliquant cette méthode circulaire, le contexte social ${ }^{2}$ n'est pas plus déterminant que les structures esthétiques vis-à-vis de la culture, donc des relations sociales. L'objet de l'étude sera de mettre en évidence des homologies et non des rapports de causalité.

Ainsi, sont prises en compte différentes données: l'idéologie de l'auteur, les conditions du marché déterminant la production et la diffusion du livre, les structures narratives (structures de l'intrigue, "figures" ou "lieux" de rhétorique, traitements linguistiques), en prenant toujours en compte qu'il existe un savoir du lecteur sur la position idéologique de l'auteur.

Les motifs justifiant une analyse structurale ne sont donc pas métaphysiques, mais bel et bien critiques, historiques et sociologiques. Eco précise qu'il n'est pas nécessaire à l'analyse sémiologique de partir d'œuvres différentes pour en extraire des constantes universelles de la communication, mais plutôt de la possibilité de plusieurs comportements constants de communication afin de circonscrire les différenciations socio-historiques de ces constantes.

D'après C.-S. Peirce on peut distinguer trois types d'arguments au sein de la logique hypothétique-déductive :

- La déduction: La règle est imposée aux faits (Chaque fois qu'il y a un feu rouge, il y a ordre de s'arrêter). La règle se justifie elle-même en temps que règle. Le raisonnement mathématique est déductif.

- L'induction: La règle résulte des faits (Chaque fois qu'il y a de la fumée, il y a du feu). La règle découle de l'observation répétée de faits réels, contingents. La démarche des sciences empiriques 
est le plus souvent inductive: en Sociologie, par exemple, on considère que ce qu'une enquête montre sur un échantillon est extrapolable à une classe entière.

- L'abduction: La règle susceptible d'expliquer un fait est à découvrir sous la forme d'une hypothèse. Il s'agit d'une hypothèse, donc d'une règle possible. La démarche abductive est très souvent à l'œuvre dans les processus de découvertes scientifiques (cf les épistémologies de K. Popper et T.-S. Kuhn) ainsi qu'en sémiotique.

Il s'agit, non pas de partir d'une hypothèse, mais d'y parvenir.

L'abduction est un procédé typique par l'intermédiaire duquel, en sémiotique, on est en mesure de prendre des décisions difficiles lorsque l'on suit des instructions ambiguës. (Eco, 1992, p. 248-249).

La démarche abductive ne présente pas le confort d'un procès déductif, elle ne s'organise pas autour d'une règle centrale qui éclairerait les faits. Au contraire plus intuitive, elle inventorie et cherche à relier, organiser des données, des signes qui lui semblent devoir découler d'une loi à advenir, mais encore inconnue.

U. Eco, s'appuyant largement sur l'œuvre de C.-S. Peirce, avait déjà comparé l'abduction à ses deux démarches cousines, la déduction et l'induction. Il propose (Eco, 1988, p. 50) ainsi trois schémas inférentiels sous la forme de la figure suivante:

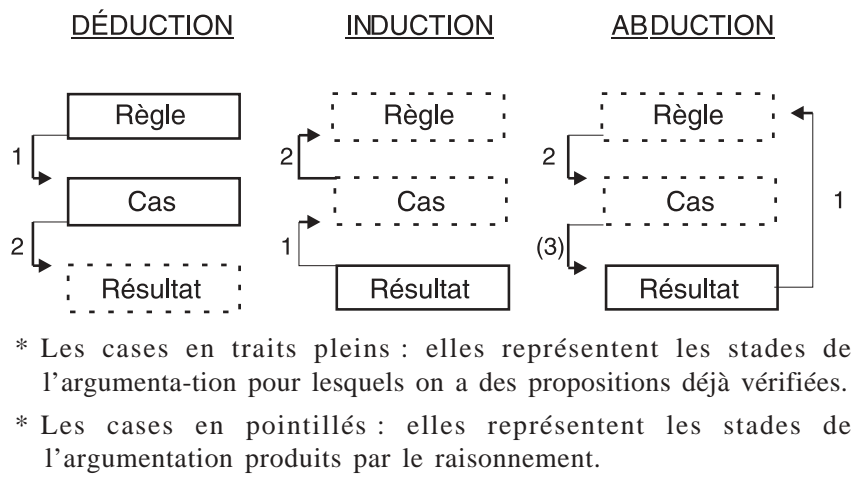


Pour U. Eco, l'abduction est une tentative pour mettre en place un système de règles de significations qui permettra à un signe d'acquérir son propre signifié. Il sera de cette manière possible de proposer une interprétation de la valeur de tel terme, tel épisode, ou tel énoncé au sein d'un texte, en s'appuyant par ailleurs sur des indices circonstanciels ou contextuels qui orienteront la structure du processus interprétatif vers l'individuation de la règle.

U.Eco (1992, p. 235-285) distingue quatre types d'abductions:

L'abduction hypercodée (ou hypothèse) dans laquelle la règle est donnée automatiquement (Eco l'appelle loi codée).

L'abduction hypocodée où il faut sélectionner une règle parmi d'autres équiprobables dans notre champ de connaissances du monde. On introduit donc une incertitude qu'il conviendra de vérifier par la suite.

L'abduction créative dans laquelle la règle doit être purement et simplement inventée.

La méta-abduction dont la mise en œuvre permet, à la suite d'une abduction créative, de décider si l'univers possible déterminé par celle-ci est isotope à l'univers de notre expérience.

Il y a des aspects esthétiques dans l'abduction créative, et ils obligent à accomplir une méta-abduction. U. Eco donne l'exemple des découvertes "révolutionnaires" qui bouleversent un paradigme scientifique établi, ${ }^{3}$ mais aussi celui de l'enquête criminelle (qu'il illustre avec les méthodes de Sherlock Holmes). Il précise ainsi:

Dans les abductions hyper et hypocodées, ce métaniveau d'inférence n'est pas indispensable, puisque nous trouvons la loi à partir d'un bagage d'expériences de mondes effectifs déjà contrôlés. Autrement dit, nous sommes autorisés par la connaissance du monde commun à penser que la loi a déjà été réputée valide (et il s'agit uniquement de savoir si c'est la bonne loi pour expliquer ces résultats). Dans les abductions créatives, on n'a pas ce type de certitude. On tend à deviner sur la nature du résultat (sa cause) ainsi que sur la nature de l'encyclopédie (de sorte que, si la nouvelle loi est vérifiée, notre 
découverte amène à un changement de paradigme). (Eco, 1992, p. 264)

Le passage de l'abduction créative à la méta-abduction est selon U. Eco (1992, p. 281) typique d'un esprit rationaliste, à l'instar du rationalisme des XVII et XVIII siècles, il cite ainsi le principe "Ordo et connexio idearum idem est ac ordo et connexio rerum" (Spinoza, Ethica, II, 7).

C'est lorsque l'on juge qu'une abduction créative est validée par une adéquation conséquente entre l'esprit et le monde extérieur que l'on entreprend une méta-abduction. U. Eco explique ainsi que, dans le cas de Sherlock Holmes, c'est une formation rationaliste qui le fait nommer "déduction" ce type de raisonnement méta-abductif qui, selon C.-S. Peirce, consiste à parier sur le résultat final sans attendre les vérifications intermédiaires. Contrairement aux détectives, les scientifiques refusent d'imposer leurs croyances comme des dogmes, U. Eco cite ainsi La vie de Galilée, de Bertolt Brecht, comme étant l'histoire de la difficulté de garder envers et contre tous ses propres abductions.

Finalement, la méta-abduction est caractérisée par le courage de défier sans vérification ultérieure la faillibilité de base qui gouverne la connaissance humaine, c'est ce que précise U. Eco (1992, p. 284):

Voilà pourquoi, dans la vie 'réelle', les détectives se trompent plus souvent (ou plus visiblement) que les scientifiques. Les détectives sont payés par la société pour leur impudence à parier métaabductivement, alors que les scientifiques sont rémunérés pour leur patience dans la vérification des abductions qu'ils font. Naturellement, pour avoir la force morale et intellectuelle de vérifier, pour demander de nouvelles vérifications et pour garder obstinément une abduction avant qu'elle ne soit définitivement vérifiée, les scientifiques aussi ont besoin de la méta-abduction.

Ce qui m'intéresse ici, c'est de souligner que la méthode proposée par U. Eco (1985) pourra aider à comprendre comment des propositions littéraires peuvent à la fois proposer une libre intervention interprétative de la part du destinataire (le lecteur), et 
présenter des caractéristiques structurales descriptibles induisant et régissant le processus des interprétations possibles. Il s'agira donc d'envisager "le phénomène de la narrativité exprimée verbalement en tant qu'interprétée par un lecteur coopérant", comme il l'explique lui-même, U. Eco prend le texte "d'en bas", à l'inverse de la démarche de A.J. Greimas qui prend le texte "d'en haut", c'est-à-dire que Eco aborde le texte à la surface de l'acte de lecture, alors que Greimas l'appréhende du plus profond de ses origines génératives.

La manifestation d'un texte consiste en une chaîne d'artifices expressifs qui attendent d'être actualisés par le destinataire; le texte est donc incomplet, à la fois parce que le texte fonctionne sur un schéma économique dépendant de la plus-value introduite par le destinataire, mais aussi parce qu'en passant de la fonction didactique à la fonction esthétique, le texte attend du lecteur qu'il l'aide à fonctionner par son initiative interprétative.

Ainsi, pour U. Eco, l'on ne constitue l'hypothèse interprétative de l'auteur qu'en se représentant le sujet d'une stratégie textuelle issue du texte étudié, et non en considérant un sujet empirique comme pensant des choses différentes de la stratégie textuelle telle qu'elle s'adresse au lecteur modèle. Ce niveau de "coopération textuelle" ne s'intéresse pas à l'actualisation des intentions du sujet empirique de l'énonciation, mais aux intentions virtuellement contenues dans l'énoncé.

La configuration de l'Auteur Modèle dépend de traces textuelles mais elle met en jeu l'univers de ce qui est derrière le texte, derrière le destinataire et probablement devant le texte et le processus de coopération (au sens où elle dépend de la question: "Qu'est-ce que je veux faire de ce texte?"). (Eco, 1985, p. 85)

Si l'on définit le travail de l'œuvre comme une re-symbolisation du donné symbolique, la première étape sociologique face à une œuvre (ici littéraire) consistera à construire, à partir de matériaux culturels, la configuration du champ symbolique. Une telle posture ouvre des perspectives sur la sociologie des représentations mais aussi éclaire la complexité de l'œuvre d'art et la multiplicité de stratégies littéraires et cognitives propres à l'œuvre. 


\section{Notes}

1 Pour le socio-anthropologue les différentes sciences humaines sont des vases communicants. Le terme de sociologie est donc employé ici dans son sens le plus strict, le plus étroit, le plus étriqué. Pour plus détails, voir: Gaudez (1997).

2 Le sociologique n'est pour moi pas plus interne à l'œuvre (au texte) qu'externe. Le social est dans et hors l'œuvre, tout comme il y a aussi du texte hors l'œuvre. Chez U. Eco, si le sociologique se situe d'une certaine manière en dehors du texte, il n'en reste pas moins que le contexte social est à la fois explicatif et raccrochable à l'œuvre dans la mesure où il est constitué d'autres textes; il y a de la textualité dans le social. En quelque sorte, il y a pour Eco une continuité entre texte et hors-texte, il ne parle pas vraiment de "contexte", mais plutôt de "co-textes".

3. Voir à ce sujet: T.-S. Kuhn (1972).

Resumo: Sobre a abdução criativa como método semioantropológico a serviço da Sociologia do Conhecimento e das representações

Uma obra de arte jamais simboliza o social ex-nihilo, ela re-simboliza. Se podemos definir o trabalho da obra como uma re-simbolização do dado simbólico, a primeira etapa sociológica em face da obra consiste em construir, a partir de materiais culturais, a configuração do campo simbólico. Uma tal postura abre perspectivas sobre a sociologia das representações além de esclarecer a complexidade da obra de arte e a multiplicidade de estratégias literárias e cognitivas próprias à obra. A descrição semiológica das estruturas da obra de arte é um dos métodos mais fecundos para recolocar a obra no seu contexto histórico-sociológico e submeter à verificação um estudo sociológico. Com apoio nos trabalhos de C.-S. Peirce e de U. Eco, trata-se aqui de indicar algumas pistas nesta direção ao se propor a abdução que, mais intuitiva do que o processo dedutivo, inventaria e busca religar, organizar os dados, os signos que lhe parecem dever resultar de uma lei que há de surgir, mas, por enquanto, não é conhecida.

Palavras-chave: abdução, obra de arte, Semiologia, Sociologia, simbólico. 


\section{Bilbiographie}

ECO, Umberto. Interprétation et surinterprétation. (1992). Paris: PUF, 1996. . Lector in fabula. Paris: Grasset, 1985. . Les limites de l'interprétation. (1990). Paris: Grasset, 1992.

KUHN, T.-S. La structure des révolutions scientifiques. (1962). Paris: Flammarion, 1972.

GAUDEZ, Florent. Pour une socio-anthropologie du texte-acteur chez Julio Cortàzar. Paris: L'Harmattan, 1997

LEENHARDT, Jacques. Une sociologie des œuvres d'art est-elle nécessaire et possible? In: MOULIN, Raymonde (Dir.). Sociologie de l'Art. (1986). Paris: L’Harmattan, 1999. p. 385-395. (Logiques sociales).

PASSERON, Jean-Claude. Le chassé-croisé des œuvres et de la sociologie. In: MOULIN, Raymonde (Dir.) Sociologie de l'Art. (1986). Paris: L'Harmattan, 1999. p. 449-459. (Logiques sociales).

WEBER, Max. L'objectivité de la connaissance dans les sciences et la politique sociales. (1904). In: . Essais sur la théorie de la science. Trad. franç. Paris: Presses Pocket, 1992. 